bibliography is provided, so it is difficult to ascertain the source of this disaster. Fortunately the other introductions are not as bad: the Kepler introduction benefits from using the Dictionary of Scientific Biography as its unacknowledged source, for example.

The most interesting part of this book is the general introduction; this is quintessential and thoughtful Hawking, clearly carrying his own stamp. He writes about the anthropic principle: "If the ultimate theory made a unique prediction for the state of the universe and its contents, it would be a remarkable coincidence that this state was in the small subset that allows life." It is almost worth the price of the book to get this quotation.

Owen Gingerich is at the Harvard-Smithsonian Center for Astrophysics, 60 Garden Street, Cambridge, Massachusetts 02138, USA.

\section{A trick of the moonlight}

The Mystery of the Moon Illusion

by Helen Ross \& Cornelis Plug

Oxford University Press: 2002. 277 pp.

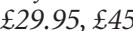

\section{Maurice Hershenson}

When the Moon is near the horizon it appears to be larger and closer than when it is high in the sky. Yet light reflected from the Moon to the eye of an observer on Earth provides the same stimulus, regardless of the elevation of the Moon. This phenomenon is known as the 'Moon illusion'. A similar illusion is observed for the setting Sun and for celestial distances between star points at different elevations.

The Moon illusion is arguably the oldest unsolved problem in modern science. It is mentioned in cuneiform script on clay tablets from the royal library of Nineveh and Babylon, dating from before the sixth century $\mathrm{BC}$, and in a collection of Chinese legends ascribed to the Taoist philosopher Lieh-tzu, dating from the fifth century BC.

Until the past hundred years or so, the illusion was thought to be a consequence of physical processes. For example, Aristotle (in the third century BC) and Ptolemy (in the second century $\mathrm{AD}$ ) incorrectly attributed the illusion to the magnifying properties of the atmosphere, and Alhazen (Ibn al-Haytham) related the illusion to the flattened appearance of the dome of the sky.

Throughout its long history, the illusion has been considered by many of the leading scientists and mathematicians of their day: da Vinci, Kepler, Descartes, Huyghens, Euler and Reimann, to name but a few. In The Mystery of the Moon Illusion, Helen Ross and Cornelis Plug carefully guide us through the history of explanations of the illusion,

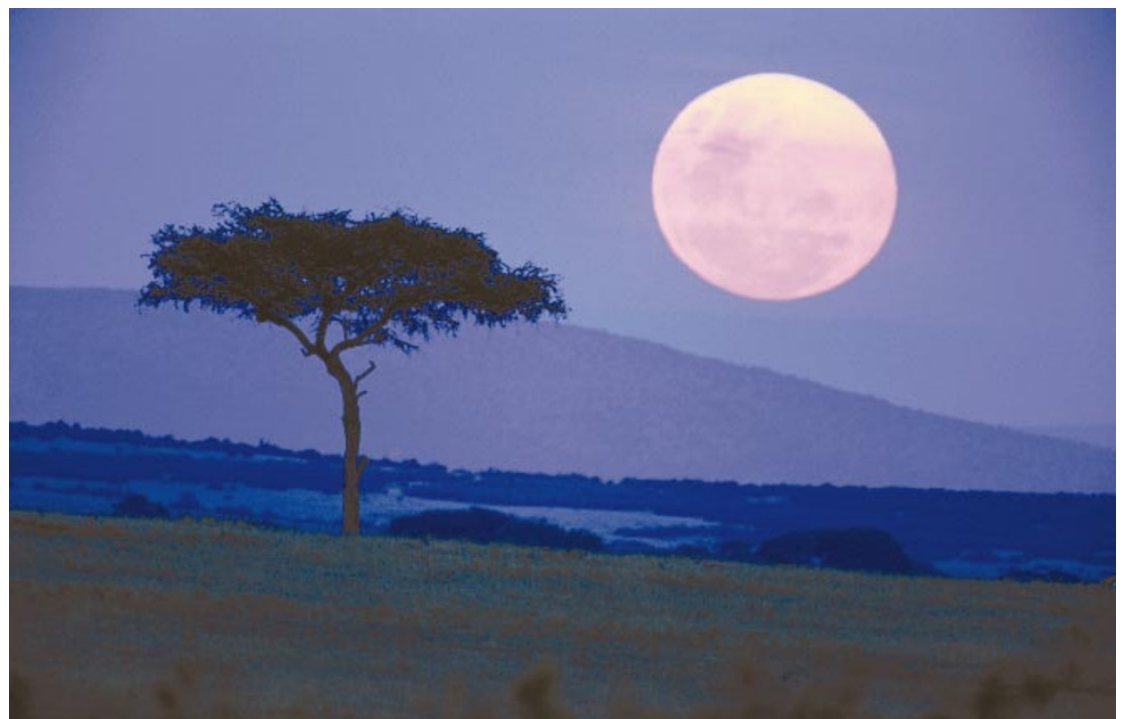

Moonrise over the savannah: but just why does the Moon appear larger when it is near the horizon?

treating in turn each aspect of the stimulus situation and its context.

Over the past century it has become clear that the Moon illusion is psychological, a consequence of the processes that underlie visual perception. Unfortunately, the authors do not provide as clear a picture of the treatment of modern explanations, perhaps because there is little agreement among current workers. For example, many modern explanations invoke the size-distance invariance hypothesis (SDIH), by which the stimulus (measured as the angular subtense of the light reaching the eye, or the visual angle) determines how large an object looks (its perceived size) and how far it appears to be from an observer (its perceived distance). The hypothesis is embodied in a psychophysical equation relating the physical stimulus (input of the visual angle) to the ratio of perceived size to perceived distance. Some theorists treat the SDIH as a fundamental law of visual perception. Indeed, it is this relationship that makes the Moon illusion such a puzzle, because it requires that the Moon looks farther away if it appears larger, and smaller if it appears closer. This is also why the illusion has sometimes been said to be paradoxical.

Modern explanations of the Moon illusion propose major changes in the form of the SDIH. For example, Walter C. Gogel and Davis L. Mertz retain the traditional form but allow perceived distance to be referenced simultaneously by different behavioural responses. Thus, the Moon may actually appear more distant to people who say: "The Moon looks close." Lloyd Kaufman and Irvin Rock's explanation is similar except that they do not include perceived distance in their formulation. Instead, they suggest that 'registered distance' (information about distance) enters the SDIH to determine perceived size. This approach alters the relationship because distance is now input information, rather than distance perception being output. Verbal statements about the perceived distance of the Moon are described as inferences based on perceived size, rather than descriptions of experience: "The Moon looks big so it must be close."

Don McCready altered the SDIH even more by substituting a perceptual outcome (the perceived visual angle) for the stimulus input (the visual angle). This proposed relationship is not just another form of the traditional psychophysical formulation, but a purely psychological relationship.

Finally, in my own explanation, the SDIH becomes a special case of a more general mechanism - a kinetic SDIH - that governs the perception of rigid object motions in depth. This mechanism automatically transforms changing stimulus inputs into objects that appear to be rigid but moving in three-dimensional space. When the stimulus does not change, as in the Moon illusion, the perceived distance of the object is determined by contextual stimulus information, such as the ground and horizon. The perceived size of the object is determined by distance information processed by the mechanism that produces rigid object motions. In this case, an object that appears close in distance, such as the Moon at the horizon, is made to appear large in size.

Despite the vast history of research into the Moon illusion, so ably reviewed by Ross and Plug, there is still no single explanation upon which even a handful of workers agree. I am left to conclude, as I did in 1989, that more research is needed, but not on the Moon illusion. An explanation of that heavenly paradox will be obvious once our understanding of visual space perception is clear. Maurice Hershenson is in the Department of Psychology, Brandeis University, Waltham, Massachusetts 02254, USA. 